\title{
VECTOR-VALUED HAUSDORFF SUMMABILITY METHODS AND ERGODIC THEOREMS
}

\author{
TAKESHI YOSHIMOTO
}

(Communicated by Paul S. Muhly)

\begin{abstract}
Suppose $X$ and $Y$ are two general Banach spaces. Let $H=\left(\Lambda_{n, k}\right)$ $(n, k=0,1,2, \ldots)$ be a general $\mathbf{B}[X, Y]$-operator valued Hausdorff summability method: $\Lambda_{n, k}=\left(\begin{array}{l}n \\ k\end{array}\right) \Delta^{n-k} U_{k}$ for $k \leq n$ and $\Lambda_{n, k}=\theta_{X, Y}$ for $k>n$, where $\left\{U_{k}\right\}_{k=0}^{\infty}$ is a sequence of operators in $\mathbf{B}[X, Y]$ and $\Delta$ denotes the backward difference (operator) and $\theta_{X, Y}(x)=0_{Y}$ (the zero element in $Y$ ) for all $x \in$ $X$. Then some necessary and sufficient conditions are given for the mean and uniform convergence of the averages
\end{abstract}

$$
\sum_{k=0}^{n}\left(\begin{array}{l}
n \\
k
\end{array}\right) \Delta^{n-k} U_{k}\left(T^{k} x\right) \quad(x \in X, T \in \mathbf{B}[X]) .
$$

\section{INTRODUCTION}

Let there be given two general Banach spaces $\left(X,\|\cdot\|_{X}\right)$ and $\left(Y,\|\cdot\|_{Y}\right)$. By $\mathbf{B}[X, Y]$ we denote, as usual, the space of bounded linear transformations from $X$ into $Y$ and $\mathbf{B}[X]=\mathbf{B}[X, X]$. Let $\left\{U_{k}\right\}_{k=0}^{\infty}$ be a sequence of operators in $\mathbf{B}[X, Y]$ and $H=\rho U \rho$ the Hausdorff method generated by $U$, where $U=\operatorname{diag}\left(U_{0}, U_{1}, \ldots\right)$ and $\rho=\left(\rho_{n, k}\right)(n, k=0,1,2, \ldots)$ is the differencing matrix given by $\rho_{n, k}=(-1)^{k}\left(\begin{array}{l}n \\ k\end{array}\right)$ for $k \leq n$ and by $\rho_{n, k}=0$ for $k>n$. A direct computation [5] then shows that $H=\left(\Lambda_{n, k}\right)(n, k=0,1,2, \ldots)$, where

$$
\begin{array}{lr}
\Lambda_{n, k}=\left(\begin{array}{l}
n \\
k
\end{array}\right) \Delta^{n-k} U_{k} & \text { if } 0 \leq k \leq n, \\
\Lambda_{n, k}=\theta_{X, Y} & \text { if } k>n .
\end{array}
$$

Here $\theta_{X, Y} \in \mathbf{B}[X, Y]$ is given by $\theta_{X, Y}(x)=0_{Y}$ (the zero element in $Y$ ) for all $x \in X$ and

$$
\begin{aligned}
\Delta U_{k} & =U_{k}-U_{k+1}, \quad \Delta^{\circ} U_{k}=U_{k}, \\
\Delta^{n} U_{k} & =\Delta \Delta^{n-1} U_{k} \quad(n=1,2, \ldots ; k=0,1,2, \ldots) .
\end{aligned}
$$

Received by the editors November 6, 1987.

1980 Mathematics Subject Classification (1985 Revision). Primary 47A35; Secondary 40G05.

Key words and phrases. Hausdorff summability method, URS-method, moment sequence, quasiregularity, mean ergodic theorem, uniform ergodic theorem. 
Following Kurtz and Tucker [6], we say that the sequence $\left\{U_{k}\right\}_{k=0}^{\infty}$ is a moment sequence in $\mathbf{B}[X, Y]$ if there is a constant $M$ independent of $\left\{x_{k}\right\}_{k=0}^{\infty}$ such that

$$
\sup _{n}\left\|\sum_{k=0}^{n}\left(\begin{array}{l}
n \\
k
\end{array}\right) \Delta^{n-k} U_{k}\left(x_{k}\right)\right\|_{Y} \leq M \cdot \sup _{k}\left\|x_{k}\right\|_{X}
$$

for every bounded sequence $\left\{x_{k}\right\}_{k=0}^{\infty}$ of points in $X$. The Hausdorff method $H=\left(\Lambda_{n, k}\right)$ will be called quasi-regular provided that

(1.1) $\left\{U_{k}\right\}_{k=0}^{\infty}$ is a moment sequence in $\mathbf{B}[X, Y]$,

(1.2) there exists an $L \in \mathbf{B}[X, Y]$ such that

$$
\sum_{k=0}^{n}\left(\begin{array}{l}
n \\
k
\end{array}\right) \Delta^{n-k} U_{k}\left(x_{k}\right) \rightarrow L(x) \quad \text { in } Y
$$

as $n \rightarrow \infty$ whenever $x_{k} \rightarrow x$ in $X$ as $k \rightarrow \infty$ for any sequence $\left\{x_{k}\right\}_{k=0}^{\infty}$ of points in $X$. In addition, if the operator $L$ in (1.2) is invertible then the method will be called strictly quasi-regular. Let $T \in \mathbf{B}[X]$. We say that the method $H=\left(\Lambda_{n, k}\right)$ is $T$-invariant if we have

$$
\text { so - } \lim _{n \rightarrow \infty} \sum_{k=0}^{n}\left(\begin{array}{l}
n \\
k
\end{array}\right) \Delta^{n-k} U_{k} \cdot T^{k+1}=\text { so }-\lim _{n \rightarrow \infty} \sum_{k=0}^{n}\left(\begin{array}{l}
n \\
k
\end{array}\right) \Delta^{n-k} U_{k} \cdot T^{k}
$$

whenever the limit on the right hand side exists. If for some $S \in \mathbf{B}[X, Y]$ we take $U_{k}=\left(\begin{array}{c}k+\alpha \\ \alpha\end{array}\right){ }^{-1} S$, where $\alpha$ is a positive integer, then the method $H=\left(\Lambda_{n, k}\right)$ becomes a vector-valued $(C, \alpha)$-method:

$$
\sum_{k=0}^{n}\left(\begin{array}{l}
n \\
k
\end{array}\right) \Delta^{n-k} U_{k}=\left(\begin{array}{c}
n+\alpha \\
\alpha
\end{array}\right)^{-1} \sum_{k=0}^{n}\left(\begin{array}{c}
n-k+\alpha-1 \\
\alpha-1
\end{array}\right) S .
$$

This $(C, \alpha$ )-method is quasi-regular (if $S$ is invertible then it is strictly quasiregular) and invariant under $T(\in \mathbf{B}[X])$ power-bounded.

Let there be given a function $K(\cdot)$ defined on $[0,1]$ with values in $\mathbf{B}\left[X, Y_{w}\right]$, where $Y_{w}$ denotes the weak sequential extension of $Y$ in the sense of Tucker [10], such that $K(\cdot)$ satisfies the Gowurin $\omega$-property and such that $K(t)$ is continuous at $t=0$ and $t=1$ with $K(0)=\theta_{X, Y_{w}}$. If we consider the averaging process

$$
\sum_{k=0}^{n}\left(\begin{array}{l}
n \\
k
\end{array}\right) \Delta^{n-k} U_{k}\left(T^{k} x\right)=\int_{0}^{1} d K(t) \sum_{k=0}^{n}\left(\begin{array}{l}
n \\
k
\end{array}\right) t^{k}(1-t)^{n-k} T^{k} x
$$

for $x \in X$ and $T \in \mathbf{B}[X]$, then the method $H=\left(\Lambda_{n, k}\right)$ determined by (1.4) is quasi-regular and invariant under $T$ power bounded. Now, for a given $T \in \mathbf{B}[X]$, the $(C, \alpha)$-mean ergodicity of $T$ always implies the possibility of the direct sum decomposition of $X$ into two subspaces $N(I-T)$ and $\overline{(I-T) X}$ (see[11, Theorem 2.1] for general URS-methods). So it seems to be an interesting problem to ask whether this fact is true for more general Hausdorff methods. 
In [7] Kurtz and Tucker gave only a sufficient condition for the mean convergence of the averages of type (1.4), that is, if $X$ is reflexive and $T \in \mathbf{B}[X]$ is power-bounded then, for any $x \in X$,

$$
\int_{0}^{1} d K(t) \cdot \sum_{k=0}^{n}\left(\begin{array}{l}
n \\
k
\end{array}\right) t^{k}(1-t)^{n-k} T^{k} x \rightarrow K(1) P x \quad \text { in } Y
$$

as $n \rightarrow \infty$, where $P$ denotes the $(C, 1)$-projection of $X$ onto the null space of $I-T$. In this paper we consider the general averaging process

$$
\sum_{k=0}^{n}\left(\begin{array}{l}
n \\
k
\end{array}\right) \Delta^{n-k} U_{k}\left(T^{k} x\right) \quad(x \in X, T \in \mathbf{B}[X])
$$

and give necessary and sufficient conditions for the mean and uniform convergence of the averages of type (1.5) under a certain restriction on the method $H=\left(\Lambda_{n, k}\right)$ (see Condition $\left.(*)\right)$. One of our results gives a partial answer to the question mentioned above. Unfortunately we do not know the complete answer to the question in the general setting without any additional conditions.

\section{Mean convergence}

The symbols $D(W), R(W)$ and $N(W)$ will be used for denoting the domain, range and null space of an operator $W$ respectively. The symbol $\mathfrak{G}(A)$ denotes the linear subspace spanned by a set $A \subset X$. It is then easily seen that $\bigcap_{m \geq 1} N\left(I-T^{m}\right)=N(I-T)$ and $\overline{\mathfrak{G}\left(\bigcup_{m \geq 1} R\left(I-T^{m}\right)\right)}=\overline{(I-T) X}$. Given a quasi-regular Hausdorff method $H=\left(\Lambda_{n, k}\right)$ and a $T \in \mathbf{B}[X]$, let

$$
Q_{T}=\text { so }-\lim _{n \rightarrow \infty} \sum_{k=0}^{n}\left(\begin{array}{l}
n \\
k
\end{array}\right) \Delta^{n-k} U_{k} \cdot T^{k}
$$

and let $P_{T}$ be a bounded linear projection of $X$ onto $N(I-T)$ with $T P_{T}=$ $P_{T} T=P_{T}$. Now we set up the following statements:

(I) $D\left(Q_{T}\right)=X$ and $Q_{T}=L P_{T}$ (with $L$ in (1.2)), i.e.,

$$
\forall x \in X, \quad Y-\lim _{n \rightarrow \infty} \sum_{k=0}^{n}\left(\begin{array}{l}
n \\
k
\end{array}\right) \Delta^{n-k} U_{k}\left(T^{k} x\right)=L P_{T} x .
$$

(II) (a) $\sup _{n \geq 0}\left\|\sum_{k=0}^{n}\left(\begin{array}{l}n \\ k\end{array}\right) \Delta^{n-k} U_{k} \cdot T^{k}\right\|_{\mathbf{B}[X, Y]}<+\infty$,

(b) so $-\lim _{n \rightarrow \infty} \sum_{k=0}^{n}\left(\begin{array}{l}n \\ k\end{array}\right) \Delta^{n-k} U_{k} \cdot T^{k}(I-T)=\theta_{X, Y}$.

(III) For each $x \in X$, there exists a subsequence $\left\{n_{i}\right\}$ of $\{n\}$ such that with $L$ in (1.2)

$$
w(Y)-\lim _{i \rightarrow \infty} \sum_{k=0}^{n_{i}}\left(\begin{array}{c}
n_{i} \\
k
\end{array}\right) \Delta^{n_{i}-k} U_{k}\left(T^{k} x\right)=L P_{T} x .
$$

(IV) $X=N(I-T) \oplus \overline{(I-T) X}$. 
Condition (*). For each $x^{*} \in X^{*}$ there exists a $y^{*} \in Y^{*}$ such that for $x_{1}, x_{2} \in$ $X$

$$
\begin{aligned}
& x^{*}\left(x_{1}\right)=x^{*}\left(x_{2}\right) \Rightarrow y^{*}\left(U_{k} x_{1}\right)=y^{*}\left(U_{k} x_{2}\right) \quad(k=0,1,2, \ldots) \\
& x^{*}\left(x_{1}\right) \neq x^{*}\left(x_{2}\right) \Rightarrow y^{*}\left(L x_{1}\right) \neq y^{*}\left(L x_{2}\right) \quad(\text { for } L \text { in }(1.2)) .
\end{aligned}
$$

If we take $X=Y$ and $S=I$ (the identity operator on $X$ ) in (1.3) then Condition $(*)$ is satisfied. If in (1.4) we take $X=Y$ and $K(t)=F(t) \cdot I$, where $F(t)$ is a real valued function of bounded variation in $0 \leq t \leq 1$ with $F(+0)=F(0)=0$ and $F(1-0)=F(1)=1$, then Condition $(*)$ is satisfied.

Theorem 2.1. Let $T \in \mathbf{B}[X]$ and let the Hausdorff method $H=\left(\Lambda_{n, k}\right)$ be strictly quasi-regular and invariant under $T$. Assume Condition $(*)$ and that there exists a projection $P_{T}$ of $X$ onto $N(I-T)$ with $P_{T}=T P_{T}=P_{T} T$. Then the following equivalence relations hold:

$$
\text { “(I)" } \quad \Leftrightarrow \quad \text { “(II) and (III)" } \quad \Leftrightarrow \quad \text { “(II) and (IV)". }
$$

The proof of this theorem will be accomplished in the following three lemmas.

Lemma 2.1. Let $T \in \mathbf{B}[X]$ and let the Hausdorff method $H=\left(\Lambda_{n, k}\right)$ be quasiregular and invariant under $T$. Then Statement (I) implies Statements (II) and (III).

Proof. Statement (II) follows from the uniform boundedness principle and the $T$-invariance of the method $H$. Statement (III) is an immediate consequence of Statement (I).

Lemma 2.2. Let $T \in B[X]$ and let the Hausdorff method $H=\left(\Lambda_{n, k}\right)$ be strictly quasi-regular and invariant under $T$. Assume Condition (*). Then Statements (II) and (III) imply Statement (IV).

Proof. Let $x \in X$. Statements (II)-(b) and (III) yield $y^{*}\left(L P_{T} x\right)=y^{*}\left(L P_{T} T x\right)$ for all $y^{*} \in Y^{*}$. Thus, $L P_{T} x=L P_{T} T x$ and $P_{T} x=P_{T} T x=T P_{T} x$ because of the invertibility of $L$. We wish to show that $x-P_{T} x \in \overline{(I-T) X}$. Suppose $x-P_{T} x \notin \overline{(I-T) X}$. Then there exists an $x_{0}^{*} \in X^{*}$ such that

$$
\begin{array}{ll}
x_{0}^{*}(z)=1 & \text { if } z \notin \overline{(I-T) X}, \\
x_{0}^{*}(z)=0 & \text { if } z \in \overline{(I-T) X} .
\end{array}
$$

Since $T^{i} x-T^{i+1} x \in(I-T) X \quad(i=0,1,2, \ldots)$, we have $x_{0}^{*}\left(T^{k} x\right)=x_{0}^{*}(x)$ for all $k=0,1,2, \ldots$. So by Condition $(*)$ there is a $y_{0}^{*} \in Y^{*}$ such that 
$y_{0}^{*}\left(U_{k}\left(T^{k} x\right)\right)=y_{0}^{*}\left(U_{k}(x)\right)$ for all $k=0,1,2, \ldots$ From this it follows that

$$
\begin{aligned}
y_{0}^{*}\left(\sum_{k=0}^{n_{i}}\left(\begin{array}{c}
n_{i} \\
k
\end{array}\right) \Delta^{n_{i}-k} U_{k}\left(T^{k} x\right)\right) & =\sum_{k=0}^{n_{i}}\left(\begin{array}{c}
n_{i} \\
k
\end{array}\right) \Delta^{n_{i}-k} y_{0}^{*}\left(U_{k}\left(T^{k} x\right)\right) \\
& =\sum_{k=0}^{n_{i}}\left(\begin{array}{c}
n_{i} \\
k
\end{array}\right) \Delta^{n_{i}-k} y_{0}^{*}\left(U_{k}(x)\right) \\
& =y_{0}^{*}\left(\sum_{k=0}^{n_{i}}\left(\begin{array}{c}
n_{i} \\
k
\end{array}\right) \Delta^{n_{i}-k} U_{k}(x)\right) .
\end{aligned}
$$

Letting $i \rightarrow \infty$ in (2.1) entails $y_{0}^{*}\left(L P_{T} x\right)=y_{0}^{*}(L x)$, and so, by Condition $(*)$, $x_{0}^{*}\left(P_{T} x\right)=x_{0}^{*}(x)$. This contradiction implies that $x-P_{T} x \in \overline{(I-T) X}$.

According to Statement (II)-(a), one sees that

$$
\left\|Q_{T}\right\|_{\mathbf{B}[X, Y]} \leq \lim _{n \rightarrow \infty}\left\|\sum_{k=0}^{n}\left(\begin{array}{l}
n \\
k
\end{array}\right) \Delta^{n-k} U_{k} \cdot T^{k}\right\|_{\mathbf{B}[X, Y]}<+\infty .
$$

Thus it follows that $D\left(Q_{T}\right)$ and $N\left(Q_{T}\right)$ are nonempty closed linear subspaces of $X$. We see from Statement (II)-(b) that $D\left(Q_{T}\left(I-T^{m}\right)\right)=X$ and $R\left(I-T^{m}\right) \subset N\left(Q_{T}\right)$ for all $m=1,2, \ldots$ Hence $\overline{(I-T) X} \subset N\left(Q_{T}\right) \subset D\left(Q_{T}\right)$. On the other hand, the invertibility of $L$ shows that $N(I-T) \cap \overline{(I-T) X}=$ $\left\{0_{X}\right\}$. Consequently, writing $x=P_{T} x+\left(x-P_{T} x\right)$, we have $X=N(I-T) \oplus$ $\overline{(I-T) X}$.

Lemma 2.3. Let $T \in \mathbf{B}[X]$ and let the Hausdorff method $H=\left(\Lambda_{n, k}\right)$ be quasiregular and invariant under $T$. Suppose there exists a projection $P_{T}$ of $X$ onto $N(I-T)$ with $P_{T} T=T P_{T}=P_{T}$. Then Statements (II) and (IV) imply Statement $(\mathbf{I})$.

Proof. Let $x \in X$. Then $x=x_{1}+x_{2}$ with $x_{1} \in N(I-T)$ and $x_{2} \in \overline{(I-T) X}$. For $x_{1}$ one gets

$$
\sum_{k=0}^{n}\left(\begin{array}{l}
n \\
k
\end{array}\right) \Delta^{n-k} U_{k}\left(T^{k} x_{1}\right) \rightarrow L P_{T} x_{1} \quad \text { in } Y
$$

as $n \rightarrow \infty$. For $x_{2}$ one sees that for any $\varepsilon>0$ there exist $u, v \in X$ such that $x_{2}=u-T u+v$, where

$$
\|v\|_{X}<\varepsilon \cdot\left(1+\sup _{n \geq 0}\left\|\sum_{k=0}^{n}\left(\begin{array}{l}
n \\
k
\end{array}\right) \Delta^{n-k} U_{k} \cdot T^{k}\right\|_{\mathbf{B}[X, Y]}\right)^{-1} .
$$

Thus by virtue of Statement (II)-(b) we have

$$
\sum_{k=0}^{n}\left(\begin{array}{l}
n \\
k
\end{array}\right) \Delta^{n-k} U_{k}\left(T^{k}(I-T) u\right) \rightarrow 0_{Y} \quad \text { in } Y
$$


as $n \rightarrow \infty$ and by $(2.3)$

$$
\left\|\sum_{k=0}^{n}\left(\begin{array}{l}
n \\
k
\end{array}\right) \Delta^{n-k} U_{k}\left(T^{k} v\right)\right\|_{Y} \leq\left(\sup _{n \geq 0}\left\|\sum_{k=0}^{n}\left(\begin{array}{l}
n \\
k
\end{array}\right) \Delta^{n-k} U_{k} \cdot T^{k}\right\|_{\mathbf{B}[X, Y]}\right) \cdot\|v\|_{X}<\varepsilon .
$$

Hence

$$
\sum_{k=0}^{n}\left(\begin{array}{l}
n \\
k
\end{array}\right) \Delta^{n-k} U_{k}\left(T^{k} x_{2}\right) \rightarrow 0_{Y} \quad \text { in } Y
$$

as $n \rightarrow \infty$. Therefore, combining the above two parts (2.2) and (2.4) implies that $D\left(Q_{T}\right)=X$ and $Q_{T}=L P_{T}$. The proof is complete.

For $T \in \mathbf{B}[X]$ let $E_{T}=$ so $-\lim _{n \rightarrow \infty} \sum_{m=1}^{\infty} a_{n, m} T^{m}$, where $\Lambda=\left(a_{n, m}\right)$ is a $T$-invariant URS-method (an infinite regular matrix satisfying the uniformity condition in the sense of Cohen [2]). We say that $T$ is strongly $H$-ergodic (resp. strongly $\Lambda$-ergodic) if $D\left(Q_{T}\right)=X$ and $Q_{T}=L P_{T}$ as in Statement (I) (resp. $\left.D\left(E_{T}\right)=X\right)$.

Corollary 2.1. Let $T \in \mathbf{B}[X]$ be power-bounded and let the Hausdorff method $H=\left(\Lambda_{n, k}\right)$ be quasi-regular and invariant under $T$. Suppose that Statement (II) holds for $T$ and the method $H$. Then the strong $\Lambda$-ergodicity of $T$ implies the strong $H$-ergodicity of $T$.

Proof. Suppose that $T$ is strongly $\Lambda$-ergodic. Then, according to [11, Theorem 2.1] (cf. $\S 4), X=N(I-T) \oplus \overline{(I-T) X}$ and there exists a projection $P_{T}$ of $X$ onto $N(I-T)$ with $P_{T}=T P_{T}=P_{T} T$. Therefore the strong $H$-ergodicity of $T$ follows at once from Lemma 2.3.

If we consider the case that $X$ is reflexive and $T \in \mathbf{B}[X]$ is power-bounded, then $T$ is strongly $\Lambda$-ergodic ([2],[12]). In this case, Statement (II) holds for $T$ and the Hausdorff method $H$ determined by (1.4) which is quasi-regular and $T$-invariant. Thus the mean ergodic theorem of Kurtz and Tucker [7] follows at once from Corollary 2.1. We do not know whether the converse statement of Corollary 2.1 holds without any additional conditions. In this connection we have:

Corollary 2.2. Let $T \in \mathbf{B}[X]$ be power-bounded. Suppose that the Hausdorff method $H=\left(\Lambda_{n, k}\right)$ is strictly quasi-regular and invariant under $T$ and that Condition (*) holds. Then the strong $H$-ergodicity of $T$ implies the strong $\Lambda$-ergodicity of $T$.

Proof. By Theorem 2.1, $X=N(I-T) \oplus \overline{(I-T) X}$. Let $x \in X$. For any $\varepsilon>0$ there exist $x_{0}, u, v \in X$ such that $x=x_{0}+(I-T) u+v, T x_{0}=x_{0}$, $\|v\|_{X}<\varepsilon \cdot\left(1+\sup _{n \geq 1} \sum_{m=1}^{\infty}\left|a_{n, m}\right|\right)^{-1}$. Then

$$
\begin{aligned}
& \sum_{m=1}^{\infty} a_{n, m} T^{m} x-\left(\sum_{m=1}^{\infty} a_{n, m}\right) x_{0} \\
& \quad=a_{n, 1} T u+\sum_{m=1}^{\infty}\left(a_{n, m+1}-a_{n, m}\right) T^{m+1} u+\sum_{m=1}^{\infty} a_{n, m} T^{m} v
\end{aligned}
$$


while, there is a number $N$ such that for all $n>N$

$$
\begin{gathered}
\left\|a_{n, 1} T u\right\|_{X}<\varepsilon \cdot\left(\sup _{m \geq 0}\left\|T^{m}\right\|_{\mathbf{B}[X]}\right) \cdot\|u\|_{X}, \\
\left\|\sum_{m=1}^{\infty}\left(a_{n, m+1}-a_{n, m}\right) T^{m+1} u\right\|_{X}<3 \varepsilon \cdot\left(\sup _{m \geq 0}\left\|T^{m}\right\|_{\mathbf{B}[X]}\right) \cdot\|u\|_{X}, \\
\left\|\sum_{m=1}^{\infty} a_{n, m} T^{m} v\right\|_{X}<\left(\sup _{n \geq 1} \sum_{m=1}^{\infty}\left|a_{n, m}\right|\right) \cdot\left(\sup _{m \geq 0}\left\|T^{m}\right\|_{\mathbf{B}[X]}\right) \cdot\|v\|_{X} .
\end{gathered}
$$

Thus we have, for all $n$ sufficiently large,

$$
\left\|\sum_{m=1}^{\infty} a_{n, m} T^{m} x-\left(\sum_{m=1}^{\infty} a_{n, m}\right) x_{0}\right\|_{X}<\varepsilon \cdot\left(\sup _{m \geq 0}\left\|T^{m}\right\|_{\mathbf{B}[X]}\right) \cdot\left(4\|u\|_{X}+1\right) .
$$

This implies $E_{T} x=x_{0}$ and hence $D\left(E_{T}\right)=X$ as was to be shown.

Theorem 2.2. Let $T \in \mathbf{B}[X]$ and let the Hausdorff method $H=\left(\Lambda_{n, k}\right)$ be strictly quasi-regular and invariant under $T$. Assume Condition (*) and that there exists a projection $P_{T}$ of $X$ onto $N(I-T)$ with $P_{T}=T P_{T}=P_{T} T$. Then Statement (I) is equivalent to Statement "(II) and (V)":

(V) $N(I-T)$ separates $N\left(I^{*}-T^{*}\right)$, where $I^{*}$ and $T^{*}$ denote the adjoint operators of $I$ and $T$ respectively.

Proof. Statement (V) is equivalent to Statement (IV). The proof of this follows exactly the same line as that of [11, Theorem 2.4]. Therefore the conclusion of the theorem follows immediately from Theorem 2.1 .

Remark 1. If $X=Y$ and $U_{k}=\left(\begin{array}{c}k+\alpha \\ \alpha\end{array}\right)^{-1} I$, where $\alpha$ is a positive integer, then the Hausdorff method $H=\left(\Lambda_{n, k}\right)$ is regarded as a real-valued $(C, \alpha)$-method. In this case, for any $T \in \mathbf{B}[X]$, each of Statements "(I)", "(II) and (III)", "(II) and (IV)" and "(II) and (V)" implies the existence of a bounded linear projection $P_{T}$ of $X$ onto $N(I-T)$ with $P_{T}=T P_{T}=P_{T} T$. Thus we see from this that Theorem 2.1 contains the mean ergodic theorems of Yosida-Kakutani [13] and Chatterji [1]. Furthermore, it is worthwhile to notice that Theorem 2.2 is a further generalization of the theorem of Sine [9].

\section{UNIFORM CONVERGENCE}

In [8], Lin gave an elementary proof of the $(C, 1)$-uniform ergodic theorem adding the condition " $(\mathrm{I}-\mathrm{T}) \mathrm{X}$ is closed" to Dunford's theorem [3]. This suggests a similar theorem for more general Hausdorff summability methods. The following theorem is a further generalization of Lin's theorem.

Theorem 3.1. Let $T \in \mathbf{B}[X]$ and let the Hausdorff method $H=\left(\Lambda_{n, k}\right)$ be $T$ invariant and strictly quasi-regular. Assume Condition $(*)$ and that there exists a projection $P_{T}$ of $X$ onto $N(I-T)$ with $P_{T}=T P_{T}=P_{T} T$ and that, with $L$ 
in (1.2),

$$
\begin{gathered}
\text { uo }-\lim _{n \rightarrow \infty} \sum_{k=0}^{n}\left(\begin{array}{l}
n \\
k
\end{array}\right) \Delta^{n-k} U_{k}=L, \\
\text { uo }-\lim _{n \rightarrow \infty} \sum_{k=0}^{n}\left(\begin{array}{l}
n \\
k
\end{array}\right) \Delta^{n-k} U_{k} \cdot T^{k}(I-T)=\theta_{X, Y} .
\end{gathered}
$$

Then the following conditions are equivalent:
(A) uo - $\lim _{n \rightarrow \infty} \sum_{k=0}^{n}\left(\begin{array}{l}n \\ k\end{array}\right) \Delta^{n-k} U_{k} \cdot T^{k}=Q_{T}$ and $Q_{T}=L P_{T}$.
(B) $X=N(I-T) \oplus(I-T) X$ and $(I-T) X$ is closed.
(C) $(I-T) X$ is closed.

Proof. (A) $\Rightarrow$ (B). In view of Theorem 2.1, it follows perforce by Condition (A) that $X=N(I-T) \oplus \overline{(I-T) X}$. Let us put $Z=\overline{(I-T) X}$. Clearly, $Z$ is invariant under $T$ and $Q_{T}=\theta_{X, Y}$ on $Z$. Thus if we denote by $T_{Z}$ the restriction of $T$ to $Z$ then

$$
\text { uo }-\lim _{n \rightarrow \infty} \sum_{k=0}^{n}\left(\begin{array}{l}
n \\
k
\end{array}\right) \Delta^{n-k} U_{k} \cdot T_{Z}^{k}=\theta_{X, Y} .
$$

Therefore, $I-T_{Z}$ is invertible on $Z$ because $L$ is so. Hence

$$
Z=\left(I-T_{Z}\right) Z=(I-T) Z \subset(I-T) X, \quad \text { and } \quad \overline{(I-T) X}=(I-T) X .
$$

(C) $\Rightarrow$ (A). Let us set $Z=(I-T) X$. By virtue of the open mapping theorem, there exists a constant $\Gamma>0$ such that for any $z \in Z$ there is a $u \in X$ with

$$
z=(I-T) u, \quad\|u\|_{X} \leq \Gamma \cdot\|z\|_{X}
$$

Therefore,

$$
\left\|\sum_{k=0}^{n}\left(\begin{array}{l}
n \\
k
\end{array}\right) \Delta^{n-k} U_{k}\left(T^{k} z\right)\right\|_{Y} \leq \Gamma \cdot\left\|\sum_{k=0}^{n}\left(\begin{array}{l}
n \\
k
\end{array}\right) \Delta^{n-k} U_{k} \cdot T^{k}(I-T)\right\|_{\mathbf{B}[X, Y]} \cdot\|z\|_{X} \cdot
$$

Note that $Z$ is invariant under $T$. Using the restriction $T_{Z}$ of $T$ to $Z$ we have, by (3.2) and (3.4),

$$
\sum_{k=0}^{n}\left(\begin{array}{l}
n \\
k
\end{array}\right) \Delta^{n-k} U_{k} \cdot T_{z}^{k} \rightarrow \theta_{X, Y} \quad \text { in } \mathbf{B}[X, Y]
$$

as $n \rightarrow \infty$. Thus $I-T_{Z}$ is invertible on $Z$ on account of the invertibility of $L$, and

$$
(I-T) X=Z=\left(I-T_{Z}\right) Z=(I-T) Z .
$$

Now, observe that $N(I-T) \cap Z=\left\{0_{X}\right\}$. Accordingly, for any $x \in X$ there is a $z \in Z$ such that $x-z \in N(I-T)$ and hence $X=N(I-T) \oplus Z$. It is 
clear that $Q_{T}=L P_{T}$ on $N(I-T)$. For $z=(I-T) u$ in (3.3) we get

$$
\left\|\sum_{k=0}^{n}\left(\begin{array}{l}
n \\
k
\end{array}\right) \Delta^{n-k} U_{k}\left(T^{k} z\right)\right\|_{Y} \leq\left\|\sum_{k=0}^{n}\left(\begin{array}{l}
n \\
k
\end{array}\right) \Delta^{n-k} U_{k} \cdot T^{k}(I-T)\right\|_{\mathbf{B}[X, Y]} \cdot\|u\|_{X} \cdot
$$

So, it follows that, for any $x \in X$,

$$
\sum_{k=0}^{n}\left(\begin{array}{l}
n \\
k
\end{array}\right) \Delta^{n-k} U_{k}\left(T^{k} x\right) \rightarrow L P_{T} x \quad \text { in } Y
$$

as $n \rightarrow \infty$. Hence $D\left(Q_{T}\right)=X$ and $Q_{T}=L P_{T}$ on $X$. Now, since $(I-T) Z$ is closed, there exists by the open mapping theorem a constant $\gamma>0$ which may depend on $T$ and $T_{Z}$ but which is independent of $x$, such that

$$
\|z\|_{X} \leq \gamma \cdot\|x\|_{X} \quad \text { whenever }(I-T) x=(I-T) z
$$

$(x \in X, z \in Z)$. Thus if for any $x \in X$ we write $x=(x-z)+z$ with $x-z \in N(I-T), z \in Z$, we obtain by (3.3) and (3.5)

$$
\begin{aligned}
& \left\|\left[\sum_{k=0}^{n}\left(\begin{array}{l}
n \\
k
\end{array}\right) \Delta^{n-k} U_{k} \cdot T^{k}-L P_{T}\right](x)\right\|_{Y} \\
& \leq(1+\gamma)\left\|\sum_{k=0}^{n}\left(\begin{array}{l}
n \\
k
\end{array}\right) \Delta^{n-k} U_{k}-L\right\|_{\mathbf{B}[X, Y]} \cdot\|x\|_{X} \\
& \quad+\gamma \cdot \Gamma \cdot\left\|\sum_{k=0}^{n}\left(\begin{array}{l}
n \\
k
\end{array}\right) \Delta^{n-k} U_{k} \cdot T^{k}(I-T)\right\|_{\mathbf{B}[X, Y]} \cdot\|x\|_{X} .
\end{aligned}
$$

This together with (3.1) and (3.2) completes the proof.

Remark 2. Let $X=Y$ in what follows. Consider a sequence $\left\{U_{n}\right\}_{n=0}^{\infty}$ of operators in $\mathbf{B}[X]$ given by $U_{n}=\mu_{n} \cdot I, n=0,1,2, \ldots$, where $\{\mu\}_{n=0}^{\infty}$ is a sequence of real numbers with $\mu_{0}=1$. The Hausdorff method $H=\left(\Lambda_{n, k}\right)$ generated by $\left\{U_{n}\right\}$ is called regular if

(i) $\sup _{n \geq 0} \sum_{k=0}^{n}\left(\begin{array}{l}n \\ k\end{array}\right)\left\|\Delta^{n-k} U_{k}\right\|_{\mathbf{B}[X]}<+\infty$;

(ii) $\left(\begin{array}{l}n \\ k\end{array}\right) \Delta^{n-k} U_{k} \rightarrow \theta_{X}$ in $\mathbf{B}[X]$ as $n \rightarrow \infty \quad(k=0,1,2, \ldots)$;

(iii) $U_{0}=I$.

For instance, if for some $a>1, b>0, p \geq 1,0<q<1$, each $\mu_{n}$ is given by

$$
\mu_{n}=\left\{\frac{\log a}{\log (n+a)}\right\}^{p} \text { or } \mu_{n}=e^{-b n^{q}}
$$

then the method $H$ is regular since each $\mu_{n}$ is a regular moment constant ([5]). If the method $H$ is regular then it is also strictly quasi-regular with $L=I$ in (1.2) and satisfies the conditions (3.1) and $(*)$. Indeed, if in (1.3) and (1.4) we take $S=I$ and $K(t)=F(t) \cdot I$, where $F(t)$ is a bounded increasing function of $t$ with $F(+0)=F(0)=0$ and $F(1-0)=F(1)=1$, then the method $H$ is regular and satisfies the condition (3.2) for every power-bounded $T \in \mathbf{B}[X]$. 
(1) Let $T \in \mathbf{B}[X]$ be power-bounded and compact. If the method $H$ is regular and $T_{\lambda}=\lambda^{-1} T$, for $(0 \neq) \lambda \in \rho(T)$, the resolvent set of $T$, satisfies the condition (3.2), then $T_{\lambda}$ is uniformly $H$-ergodic by Theorem 3.1 since $\left(I-T_{\lambda}\right) X$ is closed.

(2) Let the method $H$ be regular and let $T \in \mathbf{B}[X]$ be power-bounded and satisfy the condition (3.2). If there exist nonnegative numbers $a_{1}, \ldots, a_{N}$ with $\sum_{i=1}^{N} a_{i}=1$ such that $\left\|\sum_{i=1}^{N} a_{i} T^{i}-S\right\|_{\mathbf{B}[X]}<1$ for some compact operator $S \in \mathbf{B}[X]$, then by [8, Corollary 2], $T$ is uniformly $(C, 1)$-ergodic. Hence $(I-T) X$ is closed, and so by Theorem 3.1, $T$ is uniformly $H$-ergodic.

Now the uniform ergodic theorem has close connections with the spestral theory. To illustrate this, let $T \in \mathbf{B}[X]$ and $T_{\lambda}=\lambda^{-1} T$ for a complex number with $|\lambda|>r(T)$, where $r(T)$ stands for the spectral radius of $T$. Let $H=$ $\left(\Lambda_{n, k}\right)$ be a strictly quasi-regular Hausdorff method invariant under $T_{\lambda}$, and suppose the conditions $(*),(3.1)$ and (3.2) for $T_{\lambda}$. Suppose that there exists a projection $P_{T_{\lambda}}$ of $X$ onto $N\left(I-T_{\lambda}\right)$ with $P_{T_{\lambda}}=T_{\lambda} P_{T_{\lambda}}=P_{T_{\lambda}} T_{\lambda}$. Then Statement (A) of Theorem 3.1 applied to $T_{\lambda}$ implies that either $\lambda$ belongs to the resolvent set $\rho(T)$ or $\lambda \in \sigma(T)$ (the spectrum of $T$ ) and $\lambda$ is a pole of the resolvent $R(\mu, T)$ of order 1. In fact, by Theorem 3.1, $X=N\left(I-T_{\lambda}\right) \oplus\left(I-T_{\lambda}\right) X$ and $\left(I-T_{\lambda}\right) X$ is closed. If $N\left(I-T_{\lambda}\right)=\left\{0_{X}\right\}$ then $\left(I-T_{\lambda}\right) X=X$. Since $Q_{T_{\lambda}}$ vanishes on $\left(I-T_{\lambda}\right) X$ and $L$ in (1.2) is invertible, $I-T_{\lambda}$ is invertible and so is $\lambda I-T$. Thus $D\left((\lambda I-T)^{-1}\right)=(\lambda I-T) X=X$. On the other hand, since $r(T)<|\lambda|$, it follows that $(\lambda I-T)^{-1}=\sum_{n=0}^{\infty} T^{n} / \lambda^{n+1}$ which converges in the uniform operator topology, so that $\lambda \in \rho(T)$. If $N\left(I-T_{\lambda}\right) \neq\left\{0_{X}\right\}$ then $N(\lambda I-T) \neq\left\{0_{X}\right\}$ and $P_{\lambda}\left(=P_{T_{i}}\right)$ is non-degenerate. Hence $\lambda \in \sigma(T)$ and $(\lambda I-T) P_{\lambda}=\theta_{X}$. This implies that $\lambda$ is a pole of $R(\mu, T)$ of order 1 ([4, Theorem 18, p. 573]).

\section{URS-METHODS}

Finally we touch upon the mean and uniform convergence for real valued URS-methods. The method of proof used in $\S \S 2-3$ for the Hausdorff summability methods applies well to the case of real valued URS-methods including the $(C, \alpha)$-method with any real $\alpha>0$. Given a $T \in \mathbf{B}[X]$ and a real valued URS-method $\Lambda=\left(a_{n, m}\right) \quad(n, m=1,2, \ldots)$, we set up the following statements:

$\left(\mathbf{M} \Lambda_{1}\right) \quad$ There exists a projection $E_{T} \in \mathbf{B}[X]$ of $X$ onto $N(I-T)$, such that, for each $x \in X$,

$$
E_{T} x=\lim _{n \rightarrow \infty} \sum_{m=1}^{\infty} a_{n, m} T^{m} x, \quad E_{T}=T E_{T}=E_{T} T
$$


$\left(\mathrm{M} \Lambda_{2}\right)$
(a) $\sup _{n \geq 1}\left\|\sum_{m=1}^{\infty} a_{n, m} T^{m}\right\|_{\mathbf{B}[X]}<+\infty$,
(b) so- $\lim _{n \rightarrow \infty} \sum_{m=1}^{\infty}\left(a_{n, m+1}-a_{n, m}\right) T^{m+1}=0$.

$\left(\mathrm{M} \Lambda_{3}\right) \quad$ For each $x \in X$, the set $\left\{\sum_{m=1}^{\infty} a_{n, m} T^{m} x: n=1,2, \ldots\right\}$ is weakly sequentially compact.

$\left(\mathrm{M} \Lambda_{4}\right) \quad X=N(I-T) \oplus \overline{(I-T) X}$.

$\left(\mathrm{M} \Lambda_{5}\right) \quad N(I-T)$ separates $N\left(I^{*}-T^{*}\right)$.

Then we have the following theorem.

Theorem 4.1 (cf. [11]). Let $T \in \mathbf{B}[X]$ and let $\Lambda=\left(a_{n, m}\right)$ be a real valued $T$-invariant URS-method. Then the following equivalence relations hold:

$$
\begin{aligned}
& \text { " }\left(\mathrm{M} \Lambda_{1}\right) " \Leftrightarrow \text { " }\left(\mathrm{M} \Lambda_{2}\right) \text { and }\left(\mathrm{M} \Lambda_{3}\right) " \\
& \Leftrightarrow \text { " }\left(\mathrm{M} \Lambda_{2}\right) \text { and }\left(\mathrm{M} \Lambda_{4}\right) \text { " } \Leftrightarrow \text { " }\left(\mathrm{M} \Lambda_{2}\right) \text { and }\left(\mathrm{M} \Lambda_{5}\right) \text { ". }
\end{aligned}
$$

Moreover, by the same manner as that in $\S 3$, we can prove the following theorem.

Theorem 4.2. Let $T \in \mathbf{B}[X]$ and let $\Lambda=\left(a_{n, m}\right)$ be a real valued $T$-invariant URS-method. Suppose that Statements $\left(\mathrm{M} \Lambda_{2}\right)-(\mathrm{a})$ and $\left(M \Lambda_{2}\right)-(\mathrm{c})$ hold:

$\left(\mathrm{M} \Lambda_{2}\right)(\mathrm{c})$

$$
\text { uo }-\lim _{n \rightarrow \infty} \sum_{m=1}^{\infty}\left(a_{n, m+1}-a_{n, m}\right) T^{m+1}=0 .
$$

Then the following conditions are equivalent :

$\left(\mathrm{U} \Lambda_{1}\right) \quad$ There exists a projection $E_{T} \in \mathbf{B}[X]$ of $X$ onto $N(I-T)$ with $E_{T}=T E_{T}=E_{T} T$, such that

$$
\text { uo }-\lim _{n \rightarrow \infty}\left\|\sum_{m=1}^{\infty} a_{n, m} T^{m}-E_{T}\right\|_{\mathbf{B}[X]}=0 .
$$

$\left(\mathrm{U} \Lambda_{2}\right) \quad X=N(I-T) \oplus(I-T) X$ and $(I-T) X$ is closed.

$\left(\mathrm{U} \Lambda_{3}\right) \quad(I-T) X$ is closed.

Remark 3. Let $H=\left(\lambda_{n, k}\right)$ be a real valued Hausdorff summability method given by $\lambda_{n, k}=\left(\begin{array}{l}n \\ k\end{array}\right) \Delta^{n-k} \mu_{k}$ if $0 \leq k \leq n$ and $\lambda_{n, k}=0$ if $k>n$, where $\left\{\mu_{n}\right\}_{n=0}^{\infty}$ is a sequence of real numbers. If the method $H$ is regular and satisfies the following uniformity condition: for any $\varepsilon>0$ there exists a number $r(\varepsilon)$ such that

$$
\sum_{k=0}^{n}\left|\left(\begin{array}{l}
n+1 \\
k+1
\end{array}\right) \Delta^{n-k} \mu_{k+1}-\left(\begin{array}{c}
n+1 \\
k
\end{array}\right) \Delta^{n-k+1} \mu_{k}\right|<\varepsilon, \quad\left|\mu_{n+1}\right|<\varepsilon, n>r(\varepsilon),
$$

then it is a real valued URS-method (cf. Remarks 1 and 2). 


\section{ACKNOWLEDGMENT}

The author wishes to express his thanks to the referee for his kind advice.

\section{REFERENCES}

1. S. D. Chatterji, Les martingales et leurs applications analytiques, Lecture Notes in Math. Springer-Verlag, 307 (1973), 27-164.

2. L. W. Cohen, On the mean ergodic theorem, Ann. of Math. 41 (1940), 505-509.

3. N. Dunford, Spectral theory, I. Convergence to projections, Trans. Amer. Math. Soc. 54 (1943), 185-217.

4. N. Dunford and J. T. Schwartz, Linear operators, part 1, Interscience, New York, 1958.

5. G. H. Hardy, Divergent series, Claredon Press, Oxford, 1949.

6. L. C. Kurtz and D. H. Tucker, Vector-valued summability methods on a linear normed space, Proc. Amer. Math. Soc. 16 (1965), 419-428.

7. __, An extended form of the mean ergodic theorem, Pacific J. Math. 27 (1968), 539-545.

8. M. Lin, On the uniform ergodic theorem, Proc. Amer. Math. Soc. 43 (1974), 337-340.

9. R. Sine, A mean ergodic theorem, Proc. Amer. Math. Soc. 24 (1970), 438-439.

10. D. H. Tucker, A representation theorem for a continuous linear transformation on a space of continuous functions, Proc. Amer. Math. Soc. 16 (1965), 946-953.

11. T. Yoshimoto, Ergodic theorems and summability methods, Quart. J. Math. Oxford (2), 38 (1987), 367-379.

12. $\_$, General ergodic theorems for linear operators, Publ. I.R.M.A. Strasbourg, 376/P-208, 1988.

13. K. Yosida and S. Kakutani, Operator-theoretical treatment of Markoff's process and mean ergodic theorem, Ann. of Math. 42 (1941), 188-228.

Département de Mathématiques, Université de Strasbourg 7, Rue René-Descartes, 67084 Strasbourg Cedex, France

AND

Department of Mathematics, Toyo University, Kawagoe, Saitama 350, Japan 